\title{
NEW SOLUTIONS OF EINSTEIN EQUATIONS REPRESENTING SPINNING MASSES
}

\author{
HUMITAKA SATO \\ Research Institute for Fundamental Physics, Kyoto University, Japan \\ and
}

AKIRA TOMIMATSU

Research Institue for Theoretical Physics, Hiroshima University, Takehara, Hiroshima-ken, Japan

\begin{abstract}
We found new, stationary axisymmetric, asymptotically flat exact solutions to Einstein's vacuum field equations, which are classified by an integer $\delta$ and Kerr metric is the solution of $\delta=1$. The number of ring singularity on the equatorial plane is $\delta$. The odd $\delta$ metrices contain the surfaces of event horizon but the even $\delta$ metrices do not. Except the Kerr metric, however, the space-time becomes singular at the poles on these surfaces.
\end{abstract}

\section{References}

Tomimatsu, A. and Sato, H.: 1972 Phys. Rev. Letters 29, 1344.

Tomimatsu, A. and Sato, H.: 1973 Prog. Theor. Phys. 50, 95.

Tomimatsu, A. and Sato, H.: Lettere Nuovo Cimento, to be published. 